\title{
VIDEO-ASSISTED COMPLETION THYMECTOMY IN REFRACTORY MYASTHENIA GRAVIS
}

\author{
Tommaso Claudio Mineo, MD, ${ }^{\mathrm{a}}$ Eugenio Pompeo, MD, ${ }^{\mathrm{a}}$ Vincenzo Ambrogi, MD, ${ }^{\mathrm{a}}$ Giorgio Bernardi, MD, ${ }^{\mathrm{b}}$ \\ Cesare Iani, MD, ${ }^{\mathrm{b}}$ and Alessandro Fabrizio Sabato, $\mathrm{MD},{ }^{\mathrm{c}}$ Rome, Italy
}

Thymectomy is an effective surgical therapy complementing the medical care of patients with myasthenia gravis. Residual thymic tissue is present in $64 \%$ up to $70 \%$ of patients showing partial improvement or no response after thymectomy. ${ }^{1}$ Either it is due to a remnant of the original gland missed during the operation or it develops from ectopic thymic tissue. ${ }^{2,3}$ Completion thymectomy, carried out by the extended transsternal ${ }^{2,3}$ or the maximal transsternal-transcervical $^{4}$ approaches, has been advocated after unsuccessful transcervical thymectomy. Videoassisted thymectomy is being investigated in the belief that this procedure may also allow a complete removal of the thymus and the anterior mediastinal and lower cervical perithymic adipose tissue. ${ }^{5,6}$ We have adopted the videoassisted approach as a less invasive modality to reoperate on patients with nonthymomatous refractory myasthenia who did not undergo extended thymectomy initially.

Between December 1995 and December 1996, four patients with myasthenia gravis, three women and one man with a mean age of 41 years, underwent videoassisted completion thymectomy. The mean duration of myasthenia gravis before the initial operation was 21 months, ranging from 12 to 38 months. The initial operation was transcervical thymectomy performed by one of the authors (T.C.M.) in three patients and sternal-splitting thymectomy performed in a different institution in one. No patient underwent extended thymectomy originally. The mean interval between operations was 120 months, ranging from 72 to 180 months. Informed consent was obtained from all patients. They were given the main information regarding the different approaches and the possible complications. Worthy of note, results of preoperative computed tomography and magnetic resonance imaging were deemed negative or inconclusive regarding the identification of residual or ectopic thymus. Three patients had elevated serum titers of antibody to the acetylcholine receptor, and all had a positive edrophonium test and characteristic decreasing responses to lowfrequency repetitive nerve stimulation. At admission, ev-

From the Thoracic Surgery, Myasthenia Gravis Unit, ${ }^{a}$ and the Departments of Neurology, ${ }^{\mathrm{b}}$ and Anesthesiology, ${ }^{\mathrm{c}}$ Tor Vergata University, Rome, Italy.

Supported by a grant from MURST $60 \%$.

Received for publication August 26, 1997; accepted for publication Sept. 3, 1997.

Address for reprints: Tommaso Claudio Mineo, MD, Cattedra di Chirurgia Toracica, Universitá Tor Vergata di Roma, Ospedale S. Eugenio, P.le Umanesimo, 10-00144 Rome, Italy.

J Thorac Cardiovasc Surg 1998;115:252-4

Copyright (c) 1998 by Mosby, Inc.

$0022-5223 / 98 \$ 5.00+0 \quad \mathbf{1 2 / 5 4} / \mathbf{8 5 8 2 0}$

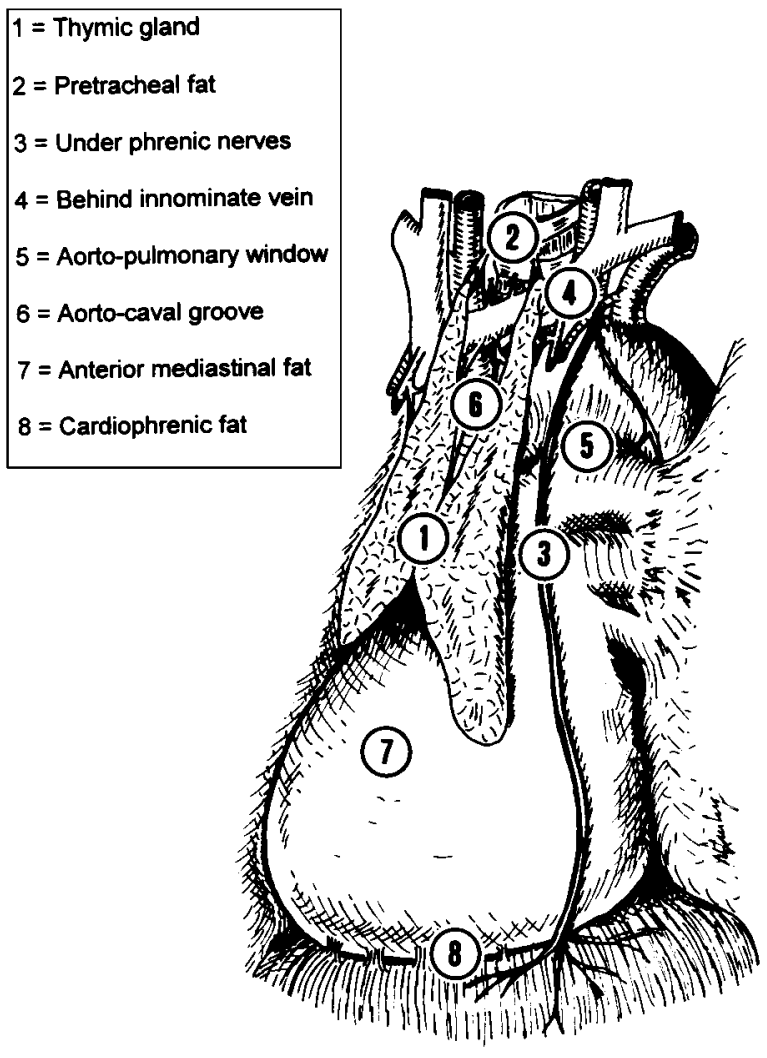

Fig. 1. Potential sites of ectopic thymic tissue that are dissected through the left thoracoscopic approach.

ery patient was completely disabled. Three patients were receiving large doses of pyridostigmine bromide (mean dose $560 \mathrm{mg} /$ day) in combination with prednisone (mean dose $45 \mathrm{mg} / \mathrm{d}$ ), and one patient was receiving prednisone and azathioprine (Table I). All attempts to taper medication were unsuccessful because of increased fatigue. Three patients were receiving long-term maintenance plasma exchange with a mean of 6.25 cycles/year (Table I). Each patient underwent four exchange cycles over the last two preoperative weeks to stabilize the clinical condition and reduce postoperative morbidity. Adjuvant pneumomediastinum was always induced within the last 24 hours to facilitate the subsequent visualization of the thymic tissue and the dissection maneuvers. ${ }^{5}$ The operation was performed after double-lumen intubation by a four-trocar access and with the patient lying in a 45-degree off-center position. Thymectomy routinely included en bloc removal of the residual thymus, if present, or all of the mediastinallower cervical perithymic adipose tissue (Fig. 1). Thymic 
Table I. Clinical data

\begin{tabular}{|c|c|c|c|c|}
\hline Variable & Case 1 & Case 2 & Case 3 & Case 4 \\
\hline Thymic tissue location & Anterior mediastinal fat & Aorto-pulmonary window & Anterior mediastinal fat & Anterior mediastinal fat \\
\hline Thymic tissue characteristics & Ectopic microscopic & Ectopic microscopic & Ectopic gross & $\begin{array}{l}\text { Residual left lower } \\
\text { horn }\end{array}$ \\
\hline $\begin{array}{l}\text { Weight of removed tissue } \\
\text { (gm); fat } \pm \text { thymus }\end{array}$ & 20 & 28 & 16 & 27 \\
\hline Follow-up (mo) & 19 & 17 & 13 & 11 \\
\hline Prednisone* (mg/day) & $50 \rightarrow 20$ & $20 \rightarrow 20$ & $50 \rightarrow 20$ & $60 \rightarrow 20$ \\
\hline Pyridostigmine* (mg/day) & $240 \rightarrow 240$ & - & $480 \rightarrow 240$ & $960 \rightarrow 480$ \\
\hline Azathioprine* $(\mathrm{mg} / \mathrm{kg} /$ day $)$ & - & $3 \rightarrow 3$ & - & - \\
\hline Plasma exchange* (cycles/yr) & $5 \rightarrow 5$ & - & $10 \rightarrow 0$ & $10 \rightarrow 0$ \\
\hline Osserman class* & $\mathrm{IIB} \rightarrow \mathrm{I}$ & $\mathrm{IIB} \rightarrow \mathrm{IIB}$ & $\mathrm{IV} \rightarrow \mathrm{IIA}$ & $\mathrm{IV} \rightarrow \mathrm{I}$ \\
\hline Outcome & Improvement & No change & Improvement & Improvement \\
\hline
\end{tabular}

*Comparison of the preoperative/postoperative data.

tissue was identified at histologic examination by the presence of Hassal's corpuscle or after immunohistochemical staining with cytokeratin and T-cell markers. The therapeutic effect of completion thymectomy was established for each patient by comparing the preoperative clinical status with the status at the most recent follow-up examination. Completion thymectomy was performed by the left video-assisted approach in three patients and by the right approach in one patient because of diffuse pleural adhesions on the left side. Mean operative time was 136 minutes, ranging from 90 to 210 minutes. In all the patients thymic remnants were found below the left innominate vein. Mean weight of the removed tissue was 22.7 gm (Table I). No operative deaths nor major morbidity occurred. Mean hospital stay was 3.25 days (range 2 to 5 days). Mean follow-up was 15.0 months (Table I). Postoperatively, one patient required further plasma exchange treatment because of a myasthenic crisis, which occurred at 6 months. At the most recent follow-up examination, although no patient was in complete remission, three had significant improvement in bulbar and limb function, needing substantially lower doses of corticosteroids and anticholinesterase drugs (Table I). Both patients who had removal of the gross thymic tissue improved after completion thymectomy.

Standard transcervical and transsternal thymectomy have been implicated as possible causes of incomplete thymectomy. ${ }^{2-4}$ In these cases completion thymectomy carried out by the extended transsternal ${ }^{2,3}$ or maximal transsternal-transcervical approaches ${ }^{4}$ proved effective in ameliorating the effects of refractory myasthenia gravis.

Similarly we noted that video-assisted completion thymectomy provided clinical improvement in three of four patients. In our experience clinical improvement clearly correlated with the reoperation, because symptoms eased soon after the operation and the improvement persisted during the follow-up period. We have always intentially performed an extended dissection of the residual thymus and adipose perithymic tissue, and we have found residual thymic tissue in all our patients.

We believe that the video-assisted approach might be considered a reliable alternative to the transsternal or the combined approach for a number of reasons. First, it is minimally invasive, which however does not seem to compromise either a complete anatomic thymectomy or a wide dissection of the anterior mediastinal and lower cervical adipose tissue. Second, patients with chronic refractory myasthenia are often receiving high doses of steroids, which exposes them to an increased risk of wound healing problems if the transsternal approach is used. Third, the transsternal reexploration of the anterior mediastinum after transcervical or transsternal thymectomy might be particularly demanding because dense adhesions might be found at the site of the innominate vein. On the other hand, the surgical reexposure of the anterior mediastinum can be facilitated by using a different route, such as the transpleural route. Finally, the magnified video imaging may allow better visualization of the vascular and nervous structures and may help reduce the risk of injuring them during the dissection maneuvers. To date the combined transsternal-transcervical thymectomy represents the gold standard in terms of extent of dissection and removal of ectopic thymic tissue in patients with myasthenia. ${ }^{4}$ However, many patients and neurologists are reluctant to accept it because of its invasiveness and because its superiority over other approaches has not yet been demonstrated. This initial experience suggests that completion thymectomy is not a contraindication for the video-assisted approach, which might prove as effective as open approaches. The role of the videoassisted thymectomy is evolving and seems to hold unsuspected potentiality. More detailed studies based on a wider accrual of patients and longer follow-up are needed to confirm these findings.

\section{REFERENCES}

1. Husain F, Ryan NJ, Hogan GR, Gonzalez E. Occurrence of invasive thymoma after thymectomy for myasthenia gravis report of a case. Neurology 1990;40:170-1.

2. Masaoka A, Monden Y, Seike Y, Tanioka T, Kagotani K. Reoperation after transcervical thymectomy for myasthenia gravis. Neurology 1982;32:83-5.

3. Miller RG, Filler-Katz A, Kiprov D, Roan R. Repeat thymec- 
tomy in chronic refractory myasthenia gravis. Neurology 1991; 41:923-4.

4. Jaretzki A, Penn AS, Younger DS, Wolff M, Olarte MR, Lovelace RE, et al. "Maximal" thymectomy for myasthenia gravis: results. J Thorac Cardiovasc Surg 1988;95:747-57.

5. Mineo TC, Pompeo E, Ambrogi V, Sabato AF, Bernardi G,
Casciani CU. Adjuvant pneumomediastinum in thoracoscopic thymectomy for myasthenia gravis. Ann Thorac Surg 1996;62: 1210-2.

6. Mack MJ, Landreneau RJ, Yim AP, Hazelrigg SR, Scruggs GR. Results of video-assisted thymectomy in patients with myasthenia gravis. J Thorac Cardiovasc Surg 1996;112:1352-60.

Thoracic Surgery Foundation for Research and Education Continuing Education Programs in Health Care Policy

The Thoracic Surgery Foundation for Research and Education invites you to attend a limited enrollment executive program on health policy, Understanding the New World of Health Care, to be held at the Kennedy School of Government, March 28-April 5, 1998. The program covers the following topics: the basics of health economics, the workings of the political environment and the media, hospital and physician reimbursement schemes, the structure of Medicare and Medicaid, the inner workings of managed care, the role of information technology, the relevant aspects of anti-trust, professional and corporate liability, and the nature and extent of health care fraud. A limited number of tuition scholarships are available. Applications for a Year-Long Sabbatical at Harvard University to do graduate studies are also available. Candidates will be accepted early on a rolling admission basis by the graduate school, and scholarships will be awarded by the Foundation's Education Committee. For more information on the Executive Program or the Year-Long Sabbatical, please call Amy Hedmark in the Foundation office at 312-644-6610. 